\title{
Exploring Multimodal Navigation Aids for Mobile Users
}

\author{
Teija Vainio \\ Tampere University of Technology, Human-Centered Technology \\ P.O. Box 589, 33101 Tampere, Finland \\ teija.vainio@tut.fi
}

\begin{abstract}
When navigating in real physical environments, as human beings we tend to display systematic or near-systematic errors with distance, direction and other navigation issues. To avoid making these errors, we choose different stratategies to find our way. While there have been a lot of HCI studies of navigation design guidelines for using maps or speech-based or tactile-based guidance in mobile devices, in this paper we introduce an initial study of multimodal navigation design utilising the design practice of episodes of motion originated from urban planning. The implications of designing cues and providing rhythm, as the design guidelines of episodes of motions suggests, are explored in this study with the subjects being pedestrians with wayfinding tasks in an urban area. The main contributions of this paper are in evaluating the design implications in the context of mobile wayfinding tasks and in reflecting the results according to human wayfinding behaviour. It is concluded that by designing predictive clues and rhythm into mobile multimodal navigation applications, we can improve navigation aids for users.
\end{abstract}

Keywords: Mobile navigation, multimodality, design.

\section{Introduction}

We must understand the nature of human wayfinding behaviour [10] such as typical navigation errors and strategies to avoid these errors to be able to understand how to support the wayfinding of mobile users. When developing tools to support wayfinding tasks of mobile users, we should pay attention that while users are using mobile wayfinding tools, usage should be consistent with user's own strategies to find a way.

Typical human navigation errors can be classified into three main categories: errors of distance, errors of direction, and other errors [25]. Errors with estimating the distance tend to be related to the amount of turns, landmarks or intersections: the more objects there are, the longer the distance estimated whereas when a mistake is related to the direction of a route, it could be caused, for example, by a person mentally rotating north-south and east-west axes, more in line with geographical axes that are on a different angle than these actual north-south axes. The third type of errors may include such issues as incorrectly estimating sizes, shapes or perspectives [25]. It is argued here that when developing novel navigation aids, the primary focus should be on supporting users to solve typical navigation errors. 
To avoid making different kinds of navigation errors, we as human beings tend to choose different types of strategies to finding our way in a real environment. Some people tend to favour a route strategy, i.e., they focus upon specific instructions on how to get from one place to another, whereas some find their way with an orientation strategy, i.e., monitoring their own position relative to reference points in the environment [14]. In recent studies related to navigation performance and wayfinding strategies it has been shown, for example, that the directions provided influence the efficiency of navigation, and the gender of the navigator is also influential [16]. Therefore, to be able to support users to avoid or to solve typical navigation errors and to develop useful navigation aids, one must understand what kind of strategies users have when making decisions how to find their way and how do gender and mode of given instructions have an effect on navigation tasks.

Although people still quite often solve their wayfinding tasks while they are on the move, either with maps printed on paper or displayed on the screen of a mobile navigation device, i.e., with a visual modality, the focus of navigation HCI research has also turned towards other human modalities, such as audio modality [2], [13] or tactile modality [3], [9]. These multimodal aids convey information through non-speech audio [10] or thematic maps [30], [32] or give feedback to visually impaired users [9] regarding how to navigate. Nonetheless, designing multimodal applications has its own challenges due to a fact that multimodal interaction occurs not only as a one-way or one-level interaction.

One key design challenge is to distinguish between when users are and are not interacting multimodally. When the focus is upon one modality at a time, it is called unimodality instead of multimodality. Furthermore, when users are interacting multimodally, recent studies have shown that users have different patterns, i.e., they interact simultaneously or they integrate their interaction sequentially [23]. However, very few studies have explored the design issues of multimodal navigation aids for mobile users by reflecting the design through typical human wayfinding behaviour such as wayfinding strategies. Hence, in order to be able to design multimodal navigation aids, one should understand the possible impacts of these factors.

It is argued here that there is no sense in designing only navigation aids based on conventional maps, see e.g. [26], and therefore more novel practices should be explored when designing navigation aids. We suggested that one possibility that can be examined is utilise design practices originating from urban planning. For several centuries, designers, urban planners and architects have developed aids for individuals navigating in real physical environments whereas, mobile HCI practitioners and researchers have studied how to support navigation with mobile devices and applications for only a few decades. Thus, for HCI professionals there are many possibilities in exploring existing design guidelines originally developed for urban planners to then support users to navigate better. Whilst there have been a lot of studies of navigation design guidelines based on Kevin Lynch's imageability [17], in this paper we propose an initial study of navigation design guidelines utilising the theory of designing episodes of motion [27].

Thus, this study investigates the relationships between two of the design principles of episodes of motion, namely the design of rhythm and cues, and users' wayfinding strategies within three different modalities: visual, audio and tactile. In this study, we define a cue as a unit of route information provided through the navigation system, 
not awareness information of others, see e.g. [20] or the environmental cues. A rhythm is defined here as route information that is provided through the navigation system regularly according period of distances.

For HCI practitioners and academics, this study presents a novel multidisciplinary design approach in order to support human navigation. In addition, for developers of mobile navigation applications, the results present an encouraging perspective in order to integrate design practices that take into account the wayfinding task in an urban environment. In addition, practical design concepts of providing cues and presenting rhythm are presented.

This paper is structured as follows. In the following section, we first present a more detailed overview of related multimodal or unimodal navigation research before presenting arguments for combining navigation design issues and urban planning practices in the section 'Designing cues and rhythm to support wayfinding'. Then, we describe the experiment and present the results. Finally, we discuss the findings and conclude the research, outlining our future work on navigation aid design.

\section{Related Work}

When exploring human navigation in a real environment with mobile applications designed to support wayfinding tasks, the focus in recent research seems to be upon issues of context (indoor, outdoor, in-vehicle, pedestrian). The user-related key concepts seem to focus upon the impact of visual ability [9] and the impact of indoor or outdoor navigation [5], as well as whether the navigators are acting as a driver [26] or as a pedestrian [14]. The main findings of non-desktop navigation research are related to interaction design, e.g., [13], [20], context [24] and adaptability, e.g., [1].

However, in mobile contexts, users' internal factors are different and external factors are dynamic and unpredictable. Furthermore, Tamminen et al. [24] argued that in relation to a mobile context, users solve navigation problems with social solutions, both national and within the mobile context, and there are temporal tensions, such as acceleration, declaration, hurrying, normal and waiting. Regarding the user interface, modality selection and interruption management are two of the main issues. [24] In relation to solving temporal tensions, it is argued here that repeating regularly navigation guidance, i.e., according certain rhythm, users are able to update their knowledge en-route.

In research on navigation tools, the tools are typically based on three major modalities: visual, audio or tactile. However, most studies are describing unimodality systems emphasizing either visual, tactile or audion modality. Recent studies related to multimodality in mobile devices have stated that multimodality can enhance the usability of mobile devices and that multimodal interaction can be superior to classical unimodal interaction in complicated tasks [14]. It has been stated also that users have a strong preference for multimodal interaction when they are interacting multimodally for a map-based task [7]. Furthermore, the research of geographic information systems (GIS) has their potential in a mobile context, for example, when personalising maps in multimodal mobile GIS [30].

Navigation research on multimodal aids has been focused upon two main streams: unimodal navigation support and characteristics of multimodality in interaction. Hence, very few studies take into account variety of wayfinding strategies, and their 
impacts on navigation performance. Furthermore, only few studies present design guidelines for mobile multimodal navigation system. This study takes another approach. It is argued here that when we are designing navigation tools to be used in real environments there are lessons to be learnt from other disciplines that have a longer tradition in designing navigation support in physical environments, i.e., urban planning and environmental psychology. Theories and practices of urban planning have been utilised in navigation, but mainly when examining navigation in virtual three-dimensional worlds [6]. Furthermore, it seems that majority of the navigation HCI research is utilizing one particular urban planning practice, i.e., Lynch's imageability [17]. We acknowledged Lynch's excellent ideas that give a solid ground also for designers in the field of navigation HCI research. However, it is argued here that there is no need to have a tunnel vision of urban planning design praxis and thereby HCI researchers and designers should extend their view beyond imageability [17] when exploring multidisciplinary design methods.

\section{Designing Cues and Rhythm to Support Wayfinding}

In some well-known urban planning design principles, such as in Lynch's [17] imageability, focusing on the design of a series of places and the design of the user's experience moving in the physical environment are emphasised. In regard to the area of designing the user's experience of moving in the physical environment, Stenros and Aura [27] presented the idea of designing episodes of motion. Concerning the experience of moving from one place to another, Stenros and Aura [27] presented the following principles for designing episodes of motion:

- Design routes so that they can be recognised as part of the entire larger system, e.g., as part of a city.

- Indicate with some kinds of clues how the route is going to continue after the episode.

- $\quad$ Design variation within a single episode to make the episode interesting.

- Design a rhythm in an episode to make it easy for users to perceive the environment

- Design open views and spaces in a route to support the user in better visualising the overall spaces.

Regarding the design principles of episodes of motion, providing cues and designing rhythm were chosen for deeper analysis in this study. These design principles are reflected through three modalities, i.e., visual, audio and tactile modalities in the wayfinding tasks of mobile pedestrians.

In this study, it was predicted that people reporting greater preference for a particular modality of guidance would be more efficient navigators. Moreover, people reporting greater preference for a route strategy would be more efficient navigators with guidance according regular rhythm. Finally, it was expected that wayfinding efficiency would vary as a function of the modality of navigation support and gender. 
This study investigates the following:

1. Two types of navigation guidance in three different modalities, namely (a) predictive cues, (b) guidance with regular rhythm within guidance provided through (i) visual, (ii) audio or (iii) tactile modality.

2. Is the most efficient and the most perceived type of guidance obtained when (a) referenced to the person's wayfinding strategy, or (b) referenced to the person's gender?

\section{Methods}

The study presented in this paper is a part of the author's $\mathrm{PhD}$ research concerning design issues of navigation aids for mobile users. In this study, both quantitative and qualitative methods were used to gather and analyse the data. Before conducting field experiments to examine multimodal navigation aids, the preliminary study identifying users' attitudes of usefulness providing two types of guidance (cues and rhythm) in three modalities (visual, audion and tactile) was carried out. The more detailed description and the results of the preliminary study are reported in our other publications, see e.g. [28]. In the field experiment impacts of two types of guidance (cues and rhythm) in three different modalities (visual, audion and tactile) in relation user's wayfinding strategies and gender issues were explored.

\subsection{Field Experiment}

The field experiment consisted of two parts. In the first part, in addition to background information, wayfinding strategies of the participants were analysed. Wayfinding strategies were studied because it is assumed that different users with different wayfinding strategies need different types of guidance. Furthermore, the participants took part in a Mental Rotation Test (MRT) with a version of Cooper and Shepard's mental rotation paradigm [18], which analysed the participants' ability to rotate objects mentally. The MRT was carried out to identify possible differences in reading visual maps among the participants.

In the second part of the field experiment, the field test was conducted in an urban area using the Wizard Of $\mathrm{Oz}$ (WOZ) technique. The field study procedure was adapted from Brewster et al. [3]. The participants were asked to complete seven wayfinding tasks and the researcher, simulating a computer, provided the navigation guidance. After each task, participants evaluated the task loads of each task subjectively. They were then interviewed after they completed all seven wayfinding tasks. During the wayfinding tasks, time of performance and number of navigation errors were recorded.

The visual modality was conducted with three types of paper maps without textual information: an overview map, maps describing part of the route and given to the participant either before the forthcoming need to turn left/right or as part of their regular rhythm. The guidance in the audio modality was given by the researcher either before the forthcoming need to turn left/right or according to the regular rhythm ("turn left/turn right/go straight forward"). The tactile guidance was given by shoulder 
tapping either before the forthcoming need to turn left/right or according to the regular rhythm by tapping on the right/left shoulder and between the shoulders.

The second part of the experiment consisted of seven wayfinding tasks (see Table 1). The routes and modalities were counterbalanced. The order of the wayfinding tasks was organised according to the principles of the Latin Square in order to exclude learning effects (see Table 1).

Table 1. The order of modalities in relation to routes and ordinal number of an experiment

\begin{tabular}{|c|c|c|c|c|c|c|c|}
\hline & 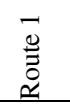 & 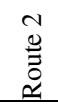 & $\begin{array}{l}\text { m } \\
\stackrel{0}{0} \\
\cong \\
\varrho\end{array}$ & $\begin{array}{l}+ \\
\stackrel{0}{\Xi} \\
\stackrel{0}{\Xi}\end{array}$ & $\begin{array}{l}n \\
\stackrel{0}{0} \\
\stackrel{0}{0}\end{array}$ & $\begin{array}{l}0 \\
\cong \\
\stackrel{0}{0} \\
\simeq\end{array}$ & 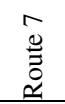 \\
\hline Visual modality: an overview map & $\begin{array}{l}1 \\
8, \\
15\end{array}$ & $\begin{array}{l}2, \\
9, \\
16\end{array}$ & $\begin{array}{l}3, \\
10 \\
17\end{array}$ & $\begin{array}{l}4, \\
11, \\
18\end{array}$ & $\begin{array}{l}5, \\
12, \\
19\end{array}$ & $\begin{array}{l}6 \\
13 \\
20\end{array}$ & $\begin{array}{l}7 \\
14, \\
21\end{array}$ \\
\hline Visual modality: maps with cues & $\begin{array}{l}4, \\
11, \\
18\end{array}$ & $\begin{array}{l}7, \\
14, \\
21\end{array}$ & $\begin{array}{l}5, \\
12, \\
19\end{array}$ & $\begin{array}{l}1, \\
8, \\
15\end{array}$ & $\begin{array}{l}6, \\
13, \\
20\end{array}$ & $\begin{array}{l}2, \\
9, \\
16\end{array}$ & $\begin{array}{l}3 \\
10 \\
17\end{array}$ \\
\hline Visual modality: maps with rhythm & $\begin{array}{l}5 \\
12, \\
19\end{array}$ & $\begin{array}{l}6 \\
13 \\
20\end{array}$ & $\begin{array}{l}7, \\
14, \\
21\end{array}$ & $\begin{array}{l}2, \\
9, \\
16\end{array}$ & $\begin{array}{l}3, \\
10 \\
17\end{array}$ & $\begin{array}{l}4 \\
11 \\
18\end{array}$ & $\begin{array}{l}1 \\
8, \\
15\end{array}$ \\
\hline Audion modality: voice with cues & $\begin{array}{l}2 \\
9, \\
16\end{array}$ & $\begin{array}{l}3, \\
10 \\
17\end{array}$ & $\begin{array}{l}6, \\
13, \\
20\end{array}$ & $\begin{array}{l}7, \\
14, \\
21\end{array}$ & $\begin{array}{l}4, \\
11, \\
18\end{array}$ & $\begin{array}{l}1, \\
8, \\
15\end{array}$ & $\begin{array}{l}5 \\
12 \\
19\end{array}$ \\
\hline Audion modality: voice with rhythm & $\begin{array}{l}7 \\
14 \\
21\end{array}$ & $\begin{array}{l}4, \\
11, \\
18\end{array}$ & $\begin{array}{l}1, \\
8, \\
15\end{array}$ & $\begin{array}{l}5, \\
12, \\
19\end{array}$ & $\begin{array}{l}2, \\
9, \\
16\end{array}$ & $\begin{array}{l}3 \\
10 \\
17\end{array}$ & $\begin{array}{l}6 \\
13, \\
20\end{array}$ \\
\hline Tactile modality: taction with cues & $\begin{array}{l}6 \\
13, \\
20\end{array}$ & $\begin{array}{l}1, \\
8, \\
15\end{array}$ & $\begin{array}{l}2, \\
9, \\
16\end{array}$ & $\begin{array}{l}3, \\
10 \\
17\end{array}$ & $\begin{array}{l}7, \\
14, \\
21\end{array}$ & $\begin{array}{l}5 \\
12 \\
19\end{array}$ & $\begin{array}{l}4 \\
11, \\
18\end{array}$ \\
\hline Tactile modality: taction with rhythm & $\begin{array}{l}3 \\
10 \\
17\end{array}$ & $\begin{array}{l}5 \\
12 \\
19\end{array}$ & $\begin{array}{l}4, \\
11, \\
18\end{array}$ & $\begin{array}{l}6, \\
13, \\
20\end{array}$ & $\begin{array}{l}1, \\
8, \\
15\end{array}$ & $\begin{array}{l}7 \\
14, \\
21\end{array}$ & $\begin{array}{l}2 \\
9, \\
16\end{array}$ \\
\hline
\end{tabular}

Each route was approximately 480 metres with four to five turns. The names of the streets were removed from the paper maps to simulate the unfamiliarity of the environment. The actual task given to the participants was to "find your way as fast as you can by walking at your normal speed". Navigation guidance was given in the form of a paper map (visual modality), with the voice (audio modality), or with haptic output (tactile modality). Furthermore, for each modality, the wayfinding instructions were given either as predicting cues or according to a regular rhythm.

While the participants were conducting the seven wayfinding tasks by walking, they were asked two questions. Examples of the questions were "What time is it?", 
"Did you notice any mailboxes on the streets?", "What kind of colours were those flowers on the left side of the street?" or "How many cars are parked in that parking area?" The aim of these interrupting questions was to simulate the context that pedestrians have when navigation is not their primary task while they are moving. Interruption management and modality selection are argued to be two key issues in mobile contexts [24]. Furthermore, the cognitive resources of mobile users are in the real contexts reserved for multiple actions simultaneously: monitoring, selecting and reacting to the context and events [20]. After each wayfinding task, the participants filled out the seven-point Nasa Task Load Index [19] to evaluate the subjective task load of each task (mental, physical and temporal demand).

After completing the tasks, participants were interviewed. Participants were asked to rank the most useful and the least useful modality for navigation support. They were then asked if they recognised any differences between the navigation support within one modality to find out if they separated predictive cues and rhythm from each other, and if they did, which one was better. Finally, they were each asked for ideas about how the multimodal navigation tool might be improved in order to be more useful.

\subsubsection{Data Analysis}

Walking times were converted to walking speed in metres per minute. The ratio of navigation performance was calculated for each participant for each mode of guidance. These ratios were used as relative indicators of performance improvement for each participant. Standard t-tests and a one-way ANOVA were performed in order to determine the significance of navigation performance for each mode of guidance. The participants' subjective evaluations of task load were used to present the most demanding and least demanding modes of guidance. Standard t-tests and a one-way analysis of variance (ANOVA) were performed in order to find possible differences.

\subsubsection{Participants}

There were 21 volunteer participants ( 14 female, 7 male) in the experiment. Of the participants, $37 \%$ were aged $18-25$ years, 32\% were aged $26-35$ years, $21 \%$ were aged $36-45$ years, and the rest of the participants $(10 \%)$ were over 46 years. Most of the participants were non-IT professionals, $40 \%$ were students and $15 \%$ were researchers. The occupations of the rest of the participants $(40 \%)$ were other than those specified. The participants were recruited by email and rewarded for their time for 15 euros.

The participants of the field experiment were not the same participants that we had in our preliminary study. Furthermore, based on the results of our preliminary study [28], we selected participants who were unfamiliar with the environment for our field experiment.

\subsubsection{Research Design}

The study involved a mixed factorial that included a between-subject comparison of individual differences in type of wayfinding strategies (orientation strategy, route strategy) and navigation performance in addition to the within-subject comparison type of navigation support (predictive cues, support with regular rhythm). 


\section{Results}

Our previous results indicate that in general, there are no significant differences between usefulness of providing cues or rhythm as a navigation aids in different modalities whereas when the environment is familiar for users, navigation aids provided with regular rhythm is not preferred as useful navigation aids .Thus, familiarity of the environment seem to be a key factor for users when evaluating usefulness of navigation aids. [28] This finding is consistent with earlier studies exploring spatial familiarity [8].

The results of the field experiment are presented according to the relative walking speed, i.e., objective data, and according to the results of experienced workload measured with the NASA Task Load Index [19], i.e., subjective data. Due to the small number of participants $(n=21)$, results based on formal statistical analysis are limited. However, they provide indications and potential trends for multimodal design.

The total number of navigation errors was 21 , and 11 of the participants made these errors. All the errors occurred when participants choose the direction, and 84\% of the errors were made with the paper maps. The most common error was confusing north and south. The remaining $16 \%$ of the errors were made with the audio modality with cues, when the participants mixed up the left and right turns. The results of Mental Rotation Test indicated no significance differences between the participants.

\subsection{Rhythms versus Cues}

After each navigation task, the participants filled out a seven-point scale NASA Task Load Index [19]. The load index was ranked for each participant to find out the tasks that participants found most stressful and having least stressful (see Table 3). According to these data, navigating with a paper-based map was the most difficult from which to find their way.

\subsubsection{Objective Data}

The average walking speeds were calculated on each route and the fastest and slowest performances (modality with predicting cues or guidance with regular rhythm) were ranked for each participant. Table 2 summarises the fastest and slowest performances. Based on analysed data, it can be argued that designing cues or rhythms in the visual modality does not improve the performance of users in wayfinding tasks. However, guidance using the audio modality with the regular rhythm seemed to be slightly better (over 21\%) than having audio with predicting cues (less than 16\%). Meanwhile, the tactile guidance predicting cues led to better performance (over 21\%) than providing guidance with a regular rhythm (see Table 2).

One-way ANOVA statistical analysis was performed and the results indicate no statistical significance between the relative walking speed and guidance mode. However, a statistically significant effect was found between gender and relative walking speed with guidance from visual rhythm, and between gender and relative walking speed with tactile guidance from rhythm $(\mathrm{p}<0.05)$.

To summarise, the results between guidance modes indicate certain trends. The main trend appears to be that the best performance was achieved by tactile guidance with predicting cues, and the worst performance was by visual guidance with predicting cues. Statistically significant results were found between gender and walking speed with guidance either from visual or tactile rhythm. 
Table 2. Relationships between relative walking speed and types of guidance

\begin{tabular}{|l|c|c|}
\hline & $\begin{array}{c}\text { Fastest } \\
\text { performance (\%) }\end{array}$ & $\begin{array}{c}\text { Slowest } \\
\text { performance (\%) }\end{array}$ \\
\hline Visual modality with an overview map & 15.8 & 21.1 \\
\hline Visual modality with predictive cues & 0 & 36.8 \\
\hline Visual modality with regular rhythm & 5.2 & 26.3 \\
\hline Audio modality with predictive cues & 15.8 & 5.2 \\
\hline Audio modality guidance with regular rhythm & 21.1 & 5.2 \\
\hline Tactile modality guidance with predictive cues & 21.1 & 0 \\
\hline Tactile modality guidance with regular rhythm & 15.8 & 5.2 \\
\hline Total & 100 & 100 \\
\hline
\end{tabular}

\subsubsection{Subjective Data}

One of the key results is that when participants were asked after completing the seven wayfinding tasks if they noticed differences between the instructions they were given in the different modalities, of the participants $60 \%$ did not remember any differences between being given predictive cues or instructions with a regular rhythm. When evaluating task load in three different modalities with two types of guidance (cues/rhythm), tactile modality with predictive cues was experienced the least stressful and an overview map the most stressful type of navigation guidance (see Table 3 ). Furthermore, a statistically significant difference $(\mathrm{p}<0.05)$ between gender and experiencing task load in audio with rhythm was found.

Table 3. Subjective estimation of the workload using the NASA Task Load Index

\begin{tabular}{|l|l|l|}
\hline & Least stressful (\%) & Most stressful (\%) \\
\hline Visual modality with an overview map & 2 & 28 \\
\hline Visual modality with predictive cues & 21 & 15 \\
\hline Visual modality with regular rhythm & 3 & 20 \\
\hline Audio modality with predictive cues & 8 & 20 \\
\hline Audio modality with regular rhythm & 20 & 15 \\
\hline Tactile modality with predictive cues & 32 & 0 \\
\hline Tactile modality with regular rhythm & 15 & 3 \\
\hline Total & 100 & 100 \\
\hline
\end{tabular}

There were no statistically significant differences in experienced task load between different modalities, but between gender and experienced task load there were statistically significant difference in audion modality with regular rhythm. 


\subsection{Impacts of Wayfinding Strategies}

In relation to our second research question, we analyze the data according the participant's wayfinding strategies. According to the participants, the least stressful guidance was the tactile modality with predictive cues regardless of the participant's wayfinding strategy (see Table 4).

Table 4. Relationships between wayfinding strategy and types of guidance

\begin{tabular}{|l|l|l|l|l|}
\hline & $\begin{array}{l}\text { Fastest } \\
\text { performance }\end{array}$ & $\begin{array}{l}\text { Slowest } \\
\text { performance }\end{array}$ & Least stressful & Most stressful \\
\hline $\begin{array}{l}\text { Participants } \\
\text { emphasising } \\
\text { orientation strategy }\end{array}$ & $\begin{array}{l}\text { Audio or tactile } \\
\text { modality with cues } \\
60 \%\end{array}$ & $\begin{array}{l}\text { Visual modality } \\
\text { with cues 40\% }\end{array}$ & $\begin{array}{l}\text { Tactile } \\
\text { modality with } \\
\text { cues 40\% }\end{array}$ & $\begin{array}{l}\text { Visual modality } \\
\text { (an overview } \\
\text { map) 45\% }\end{array}$ \\
\hline $\begin{array}{l}\text { Participants } \\
\text { emphasising route } \\
\text { strategy }\end{array}$ & $\begin{array}{l}\text { Visual, audio or } \\
\text { tactile modality } \\
\text { rhythm } \\
54 \%\end{array}$ & $\begin{array}{l}\text { Visual modality } \\
\text { with cues 45\% }\end{array}$ & $\begin{array}{l}\text { Tactile } \\
\text { modality with } \\
\text { cues 31\% }\end{array}$ & $\begin{array}{l}\text { Vision modality } \\
\text { (an overview } \\
\text { map) 34\% }\end{array}$ \\
\hline
\end{tabular}

There was a statistically significant $(\mathrm{p}<0.05)$ difference between how participants who emphasised orientation strategy evaluated task load with guidance from tactile cues. In addition, there was a statistically significant $(\mathrm{p}<0.05)$ difference between how participants who emphasised route strategy evaluated task load with guidance from tactile cues.

\section{Discussion}

Our results introduce promising design implications for the development of multimodal navigation aids to support the wayfinding of mobile users. Multidisciplinary approaches, such as design practices from urban planners, have potential when mobile human-computer interaction is investigated. In this study, the possible benefits of predictive cues or rhythm in three different modalities of navigation guidance were examined.

It is stated here that a number of important lessons from the study described in this paper can be drawn:

1. Designing cues or rhythm can be beneficial particularly when mobile users interact with tactile or audion modality. There was no clear evidence that visual cues or visual guidance with regular rhythm would be useful.

2. There is a difference between male and female navigators in relation to relative walking speed and having visual or tactile guidance with regular rhythm.

3. There is a difference between male and female navigators how participants experience the workload in audion modality with rhythm.

4. Wayfinding strategy has an impact on how human experience the workload of tactile guidance with cues

Our findings concerning the navigation performance and gender issues are consistent with earlier findings in navigation HCI studies, see e.g., [10], [15], [16]. However, 
only a few if any previous studies in the field of mobile HCI have explored the impacts of wayfinding strategies and gender issues in relation to navigation performance. Thus, besides the design practices resulting from this study and presented above, it must be noted that evaluation method that was conducted in this study has its own potential. When evaluating wayfinding tasks, the NASA Task Load Index is one suitable indicator for understanding and measuring the subjective workload, but particularly when the focus of research is on human wayfinding, wayfinding strategies should be explored.

\section{Conclusion}

This paper reported on design issues to investigate navigation performance in mobile multimodal navigation aids. The results show a good potential with designing cues and rhythm in tactile and audio guidance. In particular, the results also show that with visual modality, cues and rhythm were less beneficial than with audion or tactile modality

In the field of traffic planning, the concept of fluent traffic is useful to acknowledge when we are exploring and designing human computer interaction within mobile navigation services. Fluent navigation in the context of human computer interaction and particularly in the context of this study can be defined as easy and as smooth navigation performance as possible including relative fast walking speed with only a few navigation errors. It is also including user's subjective experience of navigation performance with minimum workload.

When providing different modalities for pedestrians to receive wayfinding instructions in urban areas, providing both predicting cues and giving instructions according to a regular rhythm should be alternatives for users to choose from. According to our results, users do not differentiate between these instructions in every case, but there are still differences when measuring the walking speed or experienced work load.

A couple of potential directions for potential future research are emerging. The first concerns studying navigation with pedestrians in more detail using some other urban planning design practice than episodes of motion to support mobile users in wayfinding. The second direction of future research could focus upon investigating how to better support different wayfinding strategies in unfamiliar environments.

In conclusion, the results show that when designing cues and rhythm into mobile navigation systems, we can support wayfinding task when pedestrians are interacting with audion or tactile modality. Furthermore, when exploring wayfinding with mobile users in general, the possible impacts of wayfinding strategies should be explored.

Acknowledgments. The author would like to acknowledge professors Kaisa Väänänen-Vainio-Mattila and Sari Kujala at the Tampere University of Technology, the partners of TOPI project at the University of Tampere, Dr Matt Jones and researcher Satu Jumisko-Pyykkö for their contributions.

\section{References}

1. Baus, J., Krüger, A., Wahlster, W.: A resource-adaptive mobile navigation system. IUI 2002. In: Proc. of the 7th International Conference on Intelligent User Interfaces (2002)

2. Baus, J., Wasinger, R., Aslan, I., Krüger, A., Maier, A., Schwartz, T.: Auditory perceptible landmarks in mobile navigation. In: Proc. of the 12th International Conference on Intelligent User Interfaces, IUI 2007 (2007) 
3. Brewster, S., Chohan, F., Brown, L.: Tactile feedback for mobile interactions. In: Proceedings of the SIGCHI Conference on Human Factors in Computing Systems. CHI 2007, San Jose, California, USA, April 28 - May 03, pp. 159-162. ACM, New York (2007)

4. Brewster, S., Lumsden, J., Bell, M., Hall, M., Tasker, S.: Multimodal 'eyes-free' interaction techniques for wearable devices. In: Proc. of the SIGCHI Conference on Human Factors in Computing Systems, pp. 473-480 (2003)

5. Butz, A., Baus, J., Krüger, A., Lohse, M.: A hybrid indoor navigation system. In: Proceedings of the 6th international Conference on intelligent User interfaces. IUI 2001, Santa Fe, New Mexico, United States, January 14-17, pp. 25-32. ACM, New York (2001)

6. Charitos, D.: Designing Space in Virtual Environments for Aiding Wayfinding Behaviour. In: Proceedings of the 4th UK Virtual Reality Special Interest Group Conference, Brunel University, Brunel University Printing Services (1997)

7. Cohen, P., McGee, D., Clow, J.: The efficiency of multimodal interaction for a map-based task. In: Proceedings of the Sixth Conference on Applied Natural Language Processing. Applied Natural Language Conferences, Seattle, Washington, April 29 - May 04, pp. 331338. Morgan Kaufmann Publishers, San Francisco (2000)

8. Gale, N., Golledge, R.G., Halperin, W.C., Couclelis, H., Niehaus, R.D.: Exploring Spatial Familiarity. The Professional Geographer 42(3), 299-313

9. Ghiani, G., Leporini, B., Paternò, F.: Vibrotactile feedback as an orientation aid for blind users of mobile guides. In: Proc. of the 10th International Conference on Human-Computer interaction with Mobile Devices and Services, pp. 431-434 (2008)

10. Golledge, R.D. (ed.): Wayfinding Behavior: Cognitive Mapping and Other Spatial Processes. John Hopkins Press, Baltimore

11. Heuten, W., Wichmann, D., Boll, S.: Interactive 3D sonification for the exploration of city maps. In: Proc. of the 4th Nordic Conference on Human-Computer interaction: Changing Roles, pp. 155-164 (2006)

12. Hoggan, E., Brewster, S.: Designing audio and tactile crossmodal icons for mobile devices. In: Proc. of the 9th International Conference on Multimodal Interfaces, pp. 162-169 (2007)

13. Holland, S., Morse, D., Gedenryd, H.: AudioGPS: Spatial Audio Navigation with a Minimal Attention Interface. Personal Ubiquitous Comput 6(4), 253-259 (2002)

14. Jöst, M., Häußler, J., Merdes, M., Malaka, R.: Multimodal interaction for pedestrians: an evaluation study. In: Proceedings of the 10th International Conference on Intelligent User Interfaces (2005)

15. Lawton, C.: Gender differences in wayfinding strategies: relationship to spatial ability and spatial anxiety. Journal of Sex Roles 30(11-12), 765-779 (1994)

16. Lawton, C.A., Kallai, J.: Gender differences in wayfinding strategies and anxiety about wayfinding: a cross-cultural comparison. Sex Roles 47, 389-401

17. Lynch, K.: Image of the City. The Technology Press \& Harvard University Press, Cambridge (1960)

18. Mental Rotation Experiment Software (MENTROT), http: //artsci.wustl.edu/ rabrams/psychlab/mentrot.htm

19. NASA Task Load Index (TLX), http://www.cc.gatech.edu/classes/AY2005/cs7470_fall/papers / TLXScale.pdf

20. Oulasvirta, A., Tamminen, S., Roto, V., Kuorelahti, J.: Interaction in 4-second bursts: the fragmented nature of attentional resources in mobile HCI. In: Proceedings of the SIGCHI Conference on Human Factors in Computing Systems. CHI 2005, Portland, Oregon, USA, April 02-07, pp. 919-928. ACM, New York (2005) 
21. Oulasvirta, A., Petit, R., Raento, M., Tiitta, S.: Interpreting and acting on mobile awareness cues. Human-Computer Interaction. Human-Computer Interaction 22(1), 97-135 (2007)

22. Oviatt, S.: Multimodal system processing in mobile environments. In: Proc. of the 13th Annual ACM Symposium on User interface Software and Technology, pp. 21-30 (2000)

23. Oviatt, S., Coulston, R., Lunsford, R.: When do we interact multimodally?: cognitive load and multimodal communication patterns. In: Proc. of the 6th International Conference on Multimodal Interfaces, pp. 129-136 (2004)

24. Tamminen, S., Oulasvirta, A., Toiskallio, K., Kankainen, A.: Understanding mobile contexts. Personal Ubiquitous Comput. 8(2), 135-143 (2004)

25. Tversky, B.: Navigating by Mind and by Body. In: Freksaet, et al. (eds.) Spatial Cognition III, pp. 1-10. Springer, Heidelberg (2003)

26. Schraggen, J.M.C.: An experimental comparison betweendifferent types of in-car navigation information (Tech. Rep. No. IZF 1991 B-1). Soesterberg, The Netherland TNO Institute for Perception (1991)

27. Stenros, H., Aura, S.: Time, Motion and Architecture. Weilin + Göös, Espoo (1987)

28. Vainio, T.: Exploring cues and rhythm for designing multimodal tools to support mobile users in way-finding. In: CHI 2009 Extended Abstracts on Human Factors in Computing Systems. CHI 2009, Boston, MA, USA, April 4-9, pp. 3715-3720. ACM, New York (2009)

29. Wahlster, W.: Smartweb: multimodal web services on the road. In: Proc. of the 15th International Conference on Multimedia, p. 16 (2007)

30. Wilson, D., Doyle, J., Weakliam, J., Bertolotto, M., Lynch, D.: Personalised maps in multimodal mobile GIS. Int. J. Web Eng. Technol. 3(2), 196-216 (2007)

31. Zhao, H.: Interactive sonification of geo-referenced data. In: CHI 2005: Extended Abstracts on Human Factors in Computing Systems, pp. 1134-1135 (2005)

32. Zhao, H., Smith, B.K., Norman, K., Plaisant, C., Shneiderman, B.: Interactive sonification of choropleth maps. IEEE MultiMedia 12(2), 26-35 (2005) 